\title{
Discovering ancestors and connecting relatives in large genomic databases
}

\author{
J. P. Nani, ${ }^{1,2 *}$ (1) L. R. Bacheller, ${ }^{3}$ J. B. Cole, ${ }^{1} \oplus$ and P. M. VanRaden ${ }^{1} \oplus$ \\ ${ }^{1}$ USDA, Agricultural Research Service, Animal Genomics and Improvement Laboratory, Beltsville, MD 20705-2350 \\ ${ }^{2}$ Estación Experimental Agropecuaria Rafaela, Instituto Nacional de Tecnología Agropecuaria, 22-2300, Rafaela, SF, Argentina \\ ${ }^{3}$ Council on Dairy Cattle Breeding, Bowie, MD 20716
}

\begin{abstract}
Genomic evaluation has improved both plant and animal breeding by allowing more accurate estimation of an individual's genetic potential. Because often only a small proportion of the population to be evaluated has been genotyped, genomic estimations rely heavily on complete pedigree information. Confirmation, discovery, and correction of parentage and connected relatives allow the creation of more complete pedigrees, which in turn increase the number of usable phenotypic records and prediction accuracy. Previous methods accounted for parent-progeny conflicts using SNP. More recently haplotype methods allowed discovery of distant relationships such as maternal grandsire (MGS) and maternal great-grandsire (MGGS) with improved accuracy. However, discovered MGS and MGGS often were not used, because no dam information was available to link them to the calf. An automated procedure to discover and fill missing maternal identification information was developed, allowing discovered MGS and MGGS to be used in imputation as well as in calculating breeding values for animals in the US dairy cattle database. An MGS was discovered for 295,136 animals with unknown dam, and the MGGS was discovered for 153,909 of these animals. A virtual maternal identification was added for animals with missing information. The effect of pedigree completion on progeny inbreeding, breeding values, and reliabilities was examined. Mean inbreeding of animals with missing maternal pedigree information was $6.69 \%$ before and $6.87 \%$ after pedigree assignment; expected future inbreeding was $7.24 \%$ before and $7.20 \%$ after assignment. Reliabilities for traditional breeding values increased from 26.6 to $32.6 \%$ for milk yield, 25.9 to $32.0 \%$ for fat yield, and 26.9 to $32.9 \%$ for protein yield; genomic reliabilities also increased slightly from 76.2 to $77.1 \%$ for milk, 76.0 to $76.9 \%$ for fat, and 76.3 to $77.3 \%$ for protein. The procedure developed for pedigree completion is a useful tool for improving accuracy
\end{abstract}

Received September 11, 2019.

Accepted October 25, 2019.

*Corresponding author: JuanoNani@gmail.com of national and international evaluations and aiding producers in making better mating decisions.

Key words: ancestry discovery, pedigree, genomics, genotype

\section{INTRODUCTION}

Discovery and correction of parentage has been one of the major benefits of genomic testing for plants, animals, and humans. In the case of humans, companies offer to identify ancestry, relatives, and ethnicity groups using chromosome segments shared by individuals (Ball et al., 2016). In cattle, parentage verification started by using blood group markers (Stormont et al., 1951), followed by the use of microsatellites in the 1990s (Heyen et al., 1997), and later was changed to a standard test using approximately 100 SNP markers (Heaton et al., 2002). Having more accurate pedigrees increased accuracy in estimating relationships and, therefore, reduced bias in estimates of inbreeding, genetic trend, sire variance, and across-country genetic evaluation (Banos et al., 2001; VanRaden et al., 2009). Complete pedigrees also allowed integration of genotyped and non-genotyped animals into a joint analysis using a pedigree-genomic relationship matrix (Legarra et al., 2009) and would reduce dependence on modeling unknown parents (Bradford et al., 2019).

The availability of affordable genotyping platforms allowed an increase in the number of genotyped animals and made pedigree discovery possible. Early studies (e.g., Weller et al., 2010) focused mainly on correcting or discovering sires, because most genotyped animals were males. However, introduction of the Illumina (San Diego, CA) Bovine3K BeadChip (3K) in 2010 resulted in more genotyped females that could be included in genomic evaluation and allowed for routine parentage checks (Wiggans et al., 2012). The increasing number of available SNP on commercial chips also has improved the accuracy of parentage discovery and correction. Because the information about relationships among individuals is more complete, effective confirmation and even discovery of more distant relatives has been possible using dense SNP chips and haplotypes 
(Kirkpatrick et al., 2011; van Kaam and Hayes, 2013; VanRaden et al., 2013).

The Council of Dairy Cattle Breeding (CDCB, Bowie, MD; https://www.uscdcb.com/) conducts the national genetic evaluation of dairy cattle in the United States and manages the largest cattle database in the world. For each calf record that enters the database, information about sire and dam is checked to ensure that a genotype is assigned to the correct animal and is consistent with pedigree information. The percentage of identification (ID) information that is validated is much higher for sires than for dams. For the 2.2 million animals genotyped as of January 2018, $97 \%$ of sires but only $39 \%$ of dams were validated (Wiggans et al., 2018). As the number of genotypes in the CDCB database grows daily, the number of animals that do not have dam ID reported also increases. Those animals cannot be linked properly to the rest of the population, and consequently their genetic predictions are less accurate.

Methods developed at USDA's Animal Genomics and Improvement Laboratory (Beltsville, MD) use haplotypes to accurately discover distant relationships, such as maternal grandsires (MGS) and maternal greatgrandsires (MGGS; VanRaden et al., 2013). Suggestions and corrections for MGS have been reported by CDCB to breed associations and nominators of animals for genomic evaluation. However, in many cases, discovered MGS and MGGS were never used in genomic evaluation because dam ID and sometimes even maternal granddam (MGD) ID were missing, which made linking MGS and MGGS information to the calf impossible.

The first objective of this study was to develop an automated procedure to discover and fill missing maternal ID numbers in the CDCB database, thus creating a virtual ID for these animals and allowing discovered MGS and MGGS to be linked for completion of pedigrees and for national and international evaluation. The second objective was to evaluate the effect of the added pedigree information on progeny inbreeding, breeding values, and reliabilities.

\section{MATERIALS AND METHODS}

As of April 2019, the CDCB database included genotypes for almost 2.9 million animals, pedigree information for more than 80 million animals, and yield phenotypes for more than 38 million cows. Mendelian inconsistencies were detected and resolved before genotypes for all loci were imputed, by combining population and pedigree haplotyping using Fortran program findhap.f90, version 3 (VanRaden, 2017). Imputation was based on 79,294 SNP and breed-specific haplotype libraries and allele frequencies for 5 breeds (Ayrshire,
Brown Swiss, Guernsey, Holstein, and Jersey), as described by VanRaden et al. (2011).

After imputation, the HAP method of VanRaden et al. (2013) was used to detect incorrect ancestors and discover missing ancestors by comparing and counting the number of haplotypes that the animal shares with a proposed relative. Haplotypes in 1 individual were evaluated by dividing each chromosome into 150-SNP segments. To find the maternal contribution after a genotyped sire was confirmed or discovered, the paternal haplotype was removed from the calf's genotype, and a match was declared if the maternal haplotype matched either of the 2 MGS haplotypes. This process was repeated for the next generation. If an MGS was confirmed or discovered, its contributing haplotype was removed from the animal's maternal haplotype to determine the MGD contribution, which was then checked against MGGS haplotypes (Figure 1).

The discovered ancestors were used to create a new and unique virtual ID for missing dams and to connect young animals with their ancestors, thus replacing the unknown-parent group value for that animal. In the same way, virtual dams with discovered sires and unknown dams also received a virtual dam if the MGGS was discovered, thus creating a virtual MGD. Before creating a virtual dam ID, known cow ID in the same herd as the calf were examined. If only 1 cow had a fresh date that matched the calf's birth date and a pedigree that matched the calf's discovered pedigree, that cow was suggested as the calf's dam. If no actual dam was discovered, a virtual dam ID was assigned sequentially.

After pedigrees were updated with the new virtual ID, inbreeding and expected future inbreeding (EFI) (Vanraden and Smith, 1999), traditional and genomic breeding values, and their reliabilities were estimated for milk, fat, and protein yields (Vanraden et al., 2014), to evaluate the effects of more complete pedigrees.

\section{RESULTS AND DISCUSSION}

\section{Initial Parent Status}

Of the 2,868,531 genotypes in the CDCB dairy cattle database used for genomic evaluation in April 2019, $88 \%$ were from females. Before the Bovine3K BeadChip became available in September 2010, only $39 \%$ of genotypes used in genomic evaluation were from females (Council on Dairy Cattle Breeding, 2019). North America (United States, Canada, and Mexico) accounted for $90 \%$ of all genotyped animals and $72 \%$ of genotyped bulls in April 2019; the other genotypes were from more than 50 other countries. Using the 2019 CDCB parent discovery and validation method of counting opposite 
homozygous SNP (Wiggans et al., 2009), the percentage of confirmed sires was $78 \%$ in April 2019, which was lower than the $88 \%$ reported for a subset of animals with 3K genotypes in September 2011 (Wiggans et al., 2012). This difference reflects the exponential growth in the number of genotypes received in recent years from many different countries, sometimes without a sire genotype in the database. The number of genotyped bulls has increased over 6-fold, from 53,788 in September 2011 to 340,906 in April 2019 (Council on Dairy Cattle Breeding, 2019). The percentage of confirmed dams was $33 \%$ in 2019, compared with $30 \%$ for the 2011 subset of animals with 3K genotypes (Wiggans et al., 2012). The number of genotyped cows was more than 32 times greater in April $2019(2,520,514)$ than in September 2011 (77,753; Council on Dairy Cattle Breeding, 2019). The percentages of conflicts for sires and dams were higher in $2019(8.6 \%$ and $1.4 \%$, respectively) than for the $20113 \mathrm{~K}$ subset $(6.9 \%$ and $0.7 \%$, respectively; Wiggans et al., 2012). The percentage of non-genotyped sires was lower in 2019 (1.3\%) than for the 2011 3K subset (2.5\%; Wiggans et al., 2012), as was percentage of non-genotyped dams (43.9\% in 2019 compared with $60.2 \%$ in 2011).

In April 2019, an alternative sire was suggested for $95 \%$ of animals with sire conflicts, $23 \%$ of animals with a non-genotyped sire, and $80 \%$ of animals without sire pedigree information; the overall mean was $83 \%$. An alternative dam was suggested for $73 \%$ of dam conflicts, $3 \%$ of non-genotyped animals, and $18 \%$ of ani- mals without dam pedigree information, with an overall mean of $9 \%$.

\section{Ancestor Discovery and Predictions}

Several programming modifications to reduce the number of comparisons made with the HAP method resulted in a substantial gain in processing speed for the discovery of MGS and MGGS compared with the previous method of Wiggans et al. (2018). Haplotype detection that included but was not limited to MGS and MGGS required $3.5 \mathrm{~h}$ with 20 processors for the entire population with 2.9 million genotypes. Wiggans et al. (2018) reported that processing took $8 \mathrm{~h}$ with 20 processors for 1.3 million Holsteins. Maternal grandsires were discovered for 295,136 animals with unknown dam, and MGGS were discovered for 153,909 of those animals having also unknown MGD. Of the animals with discovered MGS and MGGS, 98\% were females and $2 \%$ were males, from 5 different breeds (Holstein, 90.9\%; Jersey, 9.01\%; Brown Swiss, 0.07\%; Ayrshire, $0.01 \%$; and Guernsey, $0.01 \%$ ). Those animals were connected to their MGS and MGGS through virtual dams and virtual MGD. Because the modified HAP-method programming is faster and possibly more accurate than the method of Wiggans et al. (2018), new features for discovering additional relationships can be added to check all relatives in a single round of processing with little or no extra time cost. Relationships to sires, dams, paternal grandsires, full sibs, and clones can be

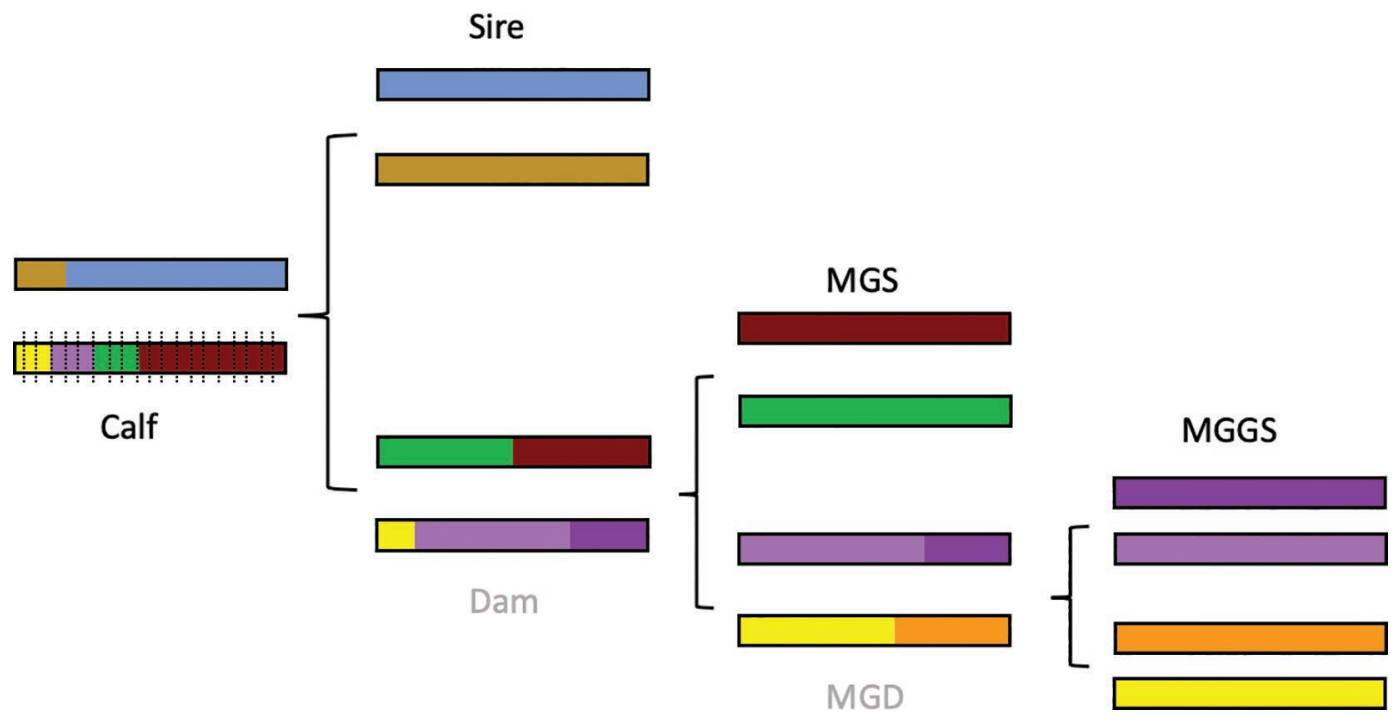

Figure 1. Schematic representation of haplotype inheritance for a chromosome. Haplotypes from 1 individual (calf) are evaluated by dividing each chromosome into 150-SNP segments (dashed vertical lines). Original haplotypes (represented by different colors) are traced back to the maternal grandsire (MGS) and maternal great-grandsire (MGGS) by removing paternal haplotype contributions. Dam and maternal granddam (MGD) contributions can then be discovered, to allow known or virtual identification information to link the calf to its ancestors. 
Table 1. Inbreeding and expected future inbreeding based on pedigree for 295,136 animals with newly found ancestors, before and after pedigree completion

\begin{tabular}{|c|c|c|c|c|}
\hline \multirow[b]{2}{*}{ Statistic } & \multicolumn{2}{|c|}{ Incomplete pedigree } & \multicolumn{2}{|c|}{ Complete pedigree } \\
\hline & $\begin{array}{l}\text { Inbreeding } \\
(\%)\end{array}$ & $\begin{array}{l}\text { Expected future inbreeding } \\
(\%)\end{array}$ & $\begin{array}{l}\text { Inbreeding } \\
(\%)\end{array}$ & $\begin{array}{l}\text { Expected future inbreeding } \\
\qquad(\%)\end{array}$ \\
\hline Minimum & 0.00 & 2.80 & 1.10 & 1.00 \\
\hline Mean & 6.69 & 7.24 & 6.87 & 7.20 \\
\hline Maximum & 16.10 & 9.40 & 32.40 & 10.00 \\
\hline
\end{tabular}

added to the discovery of MGS and MGGS procedures. The program can also verify or discover ancestors of only new individuals very quickly on a daily or weekly basis after editing and imputing their genotypes.

Pedigree Inbreeding. As expected, inbreeding and EFI changed for most (284,010 and 253,171, respectively) of the animals with a virtual dam (Table 1 ). Before pedigree completion, their inbreeding ranged from 0 to $16.1 \%$, with a mean of $6.69 \%$ (SD of 0.45 percentage points); EFI mean was $7.24 \%$ (SD of 0.56 percentage points). After parentage assignment, their mean inbreeding increased by 0.18 percentage points to $6.87 \%$ (SD of 1.59 percentage points), with a range of 1.1 to $32.4 \%$, and their EFI decreased slightly to $7.20 \%$ (SD of 0.62 percentage points). Inbreeding estimates before and after parentage assignment were statistically different $\left(P<2.2 \times 10^{-16}\right)$ and were correlated (Pearson's product-moment) by only 0.28 for an animal's own inbreeding (Spearman's rank correlation of 0.42) and by 0.90 for EFI (rank correlation of 0.88). Large changes in inbreeding were expected for these animals, because they were now connected to the rest of the animals in the pedigree. Their mean inbreeding increased because lack of information on animal ancestry often resulted in mating of highly related animals. Extreme changes in an animal's inbreeding occurred for animals with missing dam and MGD information. For example, one cow's inbreeding increased from 7.9 to $32.4 \%$. After this cow received a virtual dam and a virtual MGD, the new pedigree revealed that her sire was also her MGS.
Although EFI also changed, the effect of virtual dams and MGD was less, because EFI measures average relationship to the whole population.

Genomic Inbreeding. Mean genomic inbreeding for animals with a virtual dam or MGD based on the diagonal of the genomic relationship matrix was $5.1 \%$ (SD of 2.9 percentage points), and inbreeding ranged from $-7.8 \%$ (negative inbreeding indicated a crossbred animal) to $44.3 \%$. The mean for genomic EFI based on the off-diagonals of the genomic relationship matrix was $4.1 \%$ (SD of 0.5 percentage points). The correlation between genomic and pedigree inbreeding was 0.19 before pedigree completion and 0.57 afterward. The correlation between EFI and genomic EFI was 0.71 before pedigree completion and 0.66 afterward.

Breeding Values. Pedigree EBV for milk, fat, and protein yields (Table 2) increased by $6 \%$ on average after pedigree completion, with increases of $116 \mathrm{~kg}$ for milk, $4.5 \mathrm{~kg}$ for fat, and $3.6 \mathrm{~kg}$ for protein. Reliabilities for pedigree EBV increased by 6 percentage points for milk yield (from 26.6 to 32.6\%), 7 percentage points for fat yield (from 25.0 to $32.0 \%$ ), and 6 percentage points for protein yield (from 26.9 to $32.9 \%$ ) after adding virtual ID for dam and MGD and connecting to discovered ancestors. On average, reliabilities for pedigree EBV increased by $24 \%$ after pedigree completion.

Genomic EBV for milk, fat, and protein yields (Table 2 ) increased $3.4 \%$ on average after pedigree completion, with increases of $72 \mathrm{~kg}$ for milk, $2.7 \mathrm{~kg}$ for fat, and 2.1 $\mathrm{kg}$ for protein. Genomic reliabilities increased by $1.2 \%$

Table 2. Traditional and genomic EBV value means, SD, and reliabilities for yield traits of 295,136 animals with newly found ancestors, before and after pedigree completion

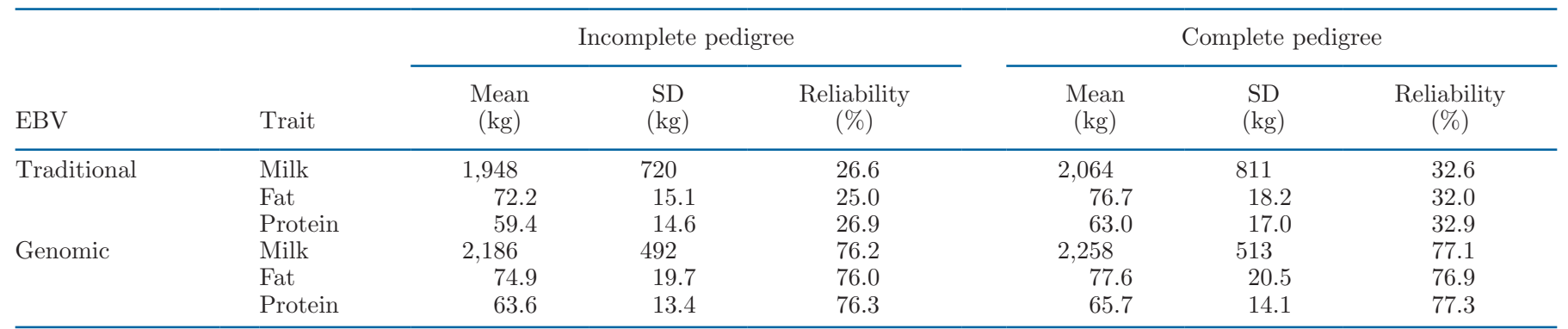


on average after pedigree completion, with increases of 0.9 percentage points for milk and fat yields (from 76.2 to $77.1 \%$ and from 76.0 to $76.9 \%$, respectively) and 1.0 percentage points for protein yield (from 76.3 to $77.3 \%$ ). Trait evaluation models remove the effect of the cow's own inbreeding but include EFI in PTA to predict inbreeding depression when mated to the current population (VanRaden, 2005). Correlations of genomic EBV with EFI included for milk, fat, and protein yields before and after pedigree completion were greater than 0.99 for all 3 traits. Mean EBV increased because parent averages for genotyped animals were higher than parent averages for the unknown-parent groups to which the genotyped animals were assigned before pedigree completion.

As numbers of genotyped animals increase, the need for optimized tools to effectively detect and fill in missing pedigree information is imperative. Because information from different sources is not always complete, genetic estimations are suboptimal. In June 2019, more than $11 \%$ of genotyped animals in the CDCB database had no dam pedigree information, but a likely candidate dam could be discovered in the pedigree database for more than $10 \%$ of the unknown dams and a unique virtual dam assigned for the remaining 90\%. Virtual dam ID could be replaced later by true dam ID if it became known, such as from foreign pedigrees that were not provided before genotyping.

To increase efficiency, calf pedigrees should be transferred from on-farm software to dairy record processing centers, and from there to CDCB before calf genotypes arrive, so that $\mathrm{CDCB}$ can confirm ancestry of calves quickly and reduce the need for discovery. Growing use of embryo transfer also increases the need for better tracking of embryos in DHI records so that recipient ID are not sent as dam ID. Timely access to complete pedigrees can improve accuracy and consistency of genomic and pedigree data.

\section{CONCLUSIONS}

Discovering MGS and MGGS enabled completion of pedigrees with missing dam information, through the discovery of genotyped dams and the use of virtual ID for dams and MGD. Linking these disconnected animals with the rest of the pedigree improved genetic and genomic predictions by correcting pedigree errors and using extra information. Identifying pedigree errors early can aid in making better pedigree- and genomeguided mating decisions. Implementing parentage discovery could reduce bias in genetic predictions for animals in unknown-parent groups and improve acrosscountry predictions.

\section{ACKNOWLEDGMENTS}

This research was supported in part by an appointment to the USDA Agricultural Research Service's Research Participation Program, which is administered by the Oak Ridge Institute for Science and Education (ORISE; Oak Ridge, TN) through an interagency agreement between the US Department of Energy and USDA (Washington, DC); ORISE is managed by Oak Ridge Associated Universities under Department of Energy contract DE-SC0014664. We acknowledge the Council on Dairy Cattle Breeding (Bowie, MD) and the Cooperative Dairy DNA Repository (Verona, WI) for providing data used in this study. Cole and VanRaden were supported by appropriated project 8042-31000002-00, "Improving Dairy Animals by Increasing Accuracy of Genomic Prediction, Evaluating New Traits, and Redefining Selection Goals," of the USDA Agricultural Research Service. Mention of trade names or commercial products in this article is solely for the purpose of providing specific information and does not imply recommendation or endorsement by the USDA. The USDA is an equal opportunity provider and employer. The authors have not stated any conflicts of interest.

\section{REFERENCES}

Ball, C. A., M. J. Barber, J. Byrnes, P. Carbonetto, K. G. Chahine, R. E. Curtis, J. M. Granka, E. Han, E. L. Hong, A. R. Kermany, N. M. Myres, K. Noto, J. Qi, K. Rand, Y. Wang, and L. Willmore. 2016. Discovering genetic matches across a massive, expanding genetic database. AncestryDNA Matching White Paper. Accessed August 15, 2019. https://www.ancestry.com/corporate/sites/ default/files/AncestryDNA-Matching-White-Paper.pdf.

Banos, G., G. R. Wiggans, and R. L. Powell. 2001. Impact of paternity errors in cow identification on genetic evaluations and international comparisons. J. Dairy Sci. 84:2523-2529. https://doi.org/10 $.3168 /$ jds.S0022-0302(01)74703-0.

Bradford, H. L., Y. Masuda, J. B. Cole, I. Misztal, and P. M. VanRaden. 2019. Modeling pedigree accuracy and uncertain parentage in single-step genomic evaluations of simulated and US Holstein datasets. J. Dairy Sci. 102:2308-2318. https://doi.org/10.3168/jds .2018-15419.

Council on Dairy Cattle Breeding. 2019. Genotypes included in evaluations by breed, chip density, presence of phenotypes (old vs. young), and evaluation year-month (cumulative). Accessed Aug. 20, 2019. https://queries.uscdcb.com/Genotype/cur_density.html.

Heaton, M. P., G. P. Harhay, G. L. Bennett, R. T. Stone, W. M. Grosse, E. Casas, J. W. Keele, T. P. L. Smith, C. G. ChitkoMcKown, and W. W. Laegreid. 2002. Selection and use of SNP markers for animal identification and paternity analysis in U.S beef cattle. Mamm. Genome 13:272-281. https://doi.org/10.1007/ s00335-001-2146-3.

Heyen, D. W., J. E. Beever, Y. Da, R. E. Everts, C. Green, H. A. Lewin, S. R. E. Bates, and J. S. Ziegle. 1997. Exclusion probabilities of 22 bovine microsatellite markers in fluorescent multiplexes for semiautomated parentage testing. Anim. Genet. 28:21-27. https://doi.org/10.1111/j.1365-2052.1997.t01-1-00057.x.

Kirkpatrick, B., S. C. Li, R. M. Karp, and E. Halperin. 2011. Pedigree reconstruction using identity by descent. J. Comput. Biol. 18:1481-1493. https://doi.org/10.1089/cmb.2011.0156. 
Legarra, A., I. Aguilar, and I. Misztal. 2009. A relationship matrix including full pedigree and genomic information. J. Dairy Sci. 92:4656-4663. https://doi.org/10.3168/jds.2009-2061.

Stormont, C., R. D. Owen, and M. R. Irwin. 1951. The B and C systems of bovine blood groups. Genetics 36:134-161.

van Kaam, J. B. C. H., and B. J. Hayes. 2013. Maternal grandsire verification and detection without imputation. Interbull Bull. 47:120-124.

VanRaden, P. M. 2005. Inbreeding adjustments and effect on genetic trend estimates. Interbull Bull. 33:81-84.

VanRaden, P. M. 2017. findhap.f90, Find haplotypes and impute genotypes using multiple chip sets and sequence data. Accessed Aug. 16, 2019. https://aipl.arsusda.gov/software/findhap/.

VanRaden, P. M., T. A. Cooper, G. R. Wiggans, J. R. O'Connell, and L. R. Bacheller. 2013. Confirmation and discovery of maternal grandsires and great-grandsires in dairy cattle. J. Dairy Sci. 96:1874-1879. https://doi.org/10.3168/jds.2012-6176.

VanRaden, P. M., J. R. O'Connell, G. R. Wiggans, and K. A. Weigel. 2011. Genomic evaluations with many more genotypes. Genet. Sel. Evol. 43:10. https://doi.org/10.1186/1297-9686-43-10.

VanRaden, P. M., and L. A. Smith. 1999. Selection and mating considering expected inbreeding of future progeny. J. Dairy Sci. 82:27712778. https://doi.org/10.3168/jds.S0022-0302(99)75534-7.

VanRaden, P. M., M. E. Tooker, J. R. Wright, C. Sun, and J. L. Hutchison. 2014. Comparison of single-trait to multi-trait national evaluations for yield, health, and fertility. J. Dairy Sci. 97:79527962. https://doi.org/10.3168/jds.2014-8489.

VanRaden, P. M., C. P. Van Tassell, G. R. Wiggans, T. S. Sonstegard, R. D. Schnabel, J. F. Taylor, and F. S. Schenkel. 2009. Invited review: Reliability of genomic predictions for North American Holstein bulls. J. Dairy Sci. 92:16-24. https://doi.org/10.3168/jds .2008-1514.

Weller, J. I., G. Glick, E. Ezra, Y. Zeron, E. Seroussi, and M. Ron. 2010. Paternity validation and estimation of genotyping error rate for the BovineSNP50 BeadChip. Anim. Genet. 41:551-553. https: //doi.org/10.1111/j.1365-2052.2010.02035.x.

Wiggans, G. R., T. A. Cooper, P. M. VanRaden, K. M. Olson, and M. E. Tooker. 2012. Use of the Illumina Bovine3K BeadChip in dairy genomic evaluation. J. Dairy Sci. 95:1552-1558. https://doi.org/ 10.3168/jds.2011-4985.

Wiggans, G. R., T. S. Sonstegard, P. M. VanRaden, L. K. Matukumalli, R. D. Schnabel, J. F. Taylor, F. S. Schenkel, and C. P. Van Tassell. 2009. Selection of single-nucleotide polymorphisms and quality of genotypes used in genomic evaluation of dairy cattle in the United States and Canada. J. Dairy Sci. 92:3431-3436. https: //doi.org/10.3168/jds.2008-1758.

Wiggans, G. R., P. M. VanRaden, and L. R. Bacheller. 2018. Methods for discovering and validating relationships among genotyped animals. Interbull Bull. 53:27-30.

\section{ORCIDS}

J. P. Nani ๑ https://orcid.org/0000-0001-7373-8055

J. B. Cole (ำ https://orcid.org/0000-0003-1242-4401

P. M. VanRaden (1) https://orcid.org/0000-0002-9123-7278 